\title{
Semi-Automatic Selection of Ground Control Points for High Resolution Remote Sensing Data in Urban Areas
}

\author{
Linda Gulbe ${ }^{1}$, Gundars Korāts ${ }^{2}$ \\ ${ }^{1,2}$ Ventspils University College, Latvia
}

\begin{abstract}
Geometrical accuracy of remote sensing data often is ensured by geometrical transforms based on Ground Control Points (GCPs). Manual selection of GCP is a time-consuming process, which requires some sort of automation. Therefore, the aim of this study is to present and evaluate methodology for easier, semi-automatic selection of ground control points for urban areas. Custom line scanning algorithm was implemented and applied to data in order to extract potential GCPs for an image analyst. The proposed method was tested for classical orthorectification and special object polygon transform. Results are convincing and show that in the test case semi-automatic methodology is able to correct locations of $70 \%$ (thermal data) $80 \%$ (orthophoto images) of buildings. Geometrical transform for subimages of approximately 3 hectares with approximately 12 automatically found GCPs resulted in RSME approximately 1 meter with standard deviation of 1.2 meters.
\end{abstract}

Keywords - Aerial photos, ground control points, semiautomatic selection.

\section{INTRODUCTION}

Geographical and geometrical accuracy is crucial for applications employing data from multiple sources. Geometrical offsets between images can introduce errors in descriptor values and burden data analysis. Typically, such problems are solved by the orthorectification procedure based on Ground Control Points (GCPs), which in fact are well defined and easily recognisable features that can be located accurately in all data sets [1]. In urban areas, the corners of buildings taken as GCPs can serve as qualitative features because these are clearly distinguishable, stable in time and available in electronic maps (both free of charge data and high quality maps managed by government institutions). GCPs are used to find coefficients for transformation equations, which define relationship between an original coordinate grid of data and corrected coordinate grid. One data source is selected as reference data, the other is corrected according to geometrical information in reference data. Spatial distribution of GCPs is crucial for ensuring accuracy of orthorectification [2]; therefore, dense and uniformly distributed GCPs are highly required. Also a number of GCPs should be larger than a minimum number of points required by the transformation function and should be as large as possible [3]. GCP manual selection by hand is a time-consuming process requiring ongoing attention; therefore, semi-automatic procedures yield an opportunity to process a large amount of data much faster and more accurately. In this context, semi-automatic procedures are solutions for selecting potential GCPs after performing the data analysis, whereas an image analyst must correct only wrong GCPs or provide GCPs in complex areas.

\section{A. Statement of the Problem}

Motivation of this study lies in the automatic analysis of roofs in the urban area to examine thermal characteristics of each roof. The proposed system for analysis reads in vector layer of buildings and extracts different raster statistics and descriptors for each building polygon. To acquire valid descriptors, accurate contours of building rooftops are required.

One approach to solving this problem could be building detection using computer vision and remote sensing data processing methods. However, this task is very challenging due to the complex structure of buildings as well as similar spectral features with other urban objects [4]. Therefore, in this study the advantages and disadvantages of geometrical correction were evaluated with semi-automatically found ground control points for vector maps and different types of aerial photos to ensure higher geometrical accuracy.

The aim of this study is to present and evaluate methodology for easier, semi-automatic selection of ground control points for urban areas.

Building corners are potential GCPs in this study and the proposed methodology aims at finding these corners in vector maps and different types of remote sensing data by seeking linear features in the images. Geometrical correction using GCPs was performed in two different ways: correction was applied to each polygon in vector map separately and standard orthorectification procedure with second order polynomial transformation model was applied to remote sensing data.

\section{B. Related Research}

Geometrical correction can be implemented by performing the following general steps [5]:

1. feature extraction;

2. feature matching;

3. transformation model estimation;

4. image registration or orthorectification.

Automation of the process can be achieved by replacing manual work of human interpreter in first two steps by automated solutions [5].

Both area-based and feature-based techniques can be used in feature extraction and matching phase. In the area-based methods, a small window in the image to be corrected is 
compared statistically with subregions in the reference image. Similarity often is measured by a normalized correlation coefficient. Liu et al. [6] presents a methodology based on assumption that potential GCRs (ground control regions) are located at pixels with higher contrast of grayscale values. These algorithms can be used to coregister images from the same source and therefore are not of the interest in this study.

Feature-based methods are more robust and mostly related to object properties such as boundary and edge features, which are less affected by data acquisition technique.

Since spectral characteristics of buildings are not particularly unique, edge detection techniques are a suggestible solution to extract linear features such as building contour elements. Some typical solutions are gradient based edge detection, Hough transform, template matching and dynamic programming [7]. Modifications of basic gradient operators, for example, Sobel operator can achieve higher edge detection accuracy [8]. Edges can be classified in three types: edge caused by surface normal discontinuities, caused by depth discontinuities, caused solely by intensity discontinuities [9]. For GCP selection only the first type of edges is of interest; however, edge detectors more or less are sensitive to all types of edges. Centres of gravity of closed boundaries, salient points and crossing points of linear features can be used as GCPs.

Many studies propose using scale invariant feature transform (SIFT) method. Ye and Shan [10] and Yu et al. [11] employed SIFT together with Harris corner detector to register multispectral satellite images such as Quickbird, WorldView, SPOT5, SPOT4 and Landsat TM. The proposed research highlights the ability of SIFT to extract sufficient amount of GCPs, despite the fact that it gives poor performance and quality when applied to multisource remote sensing data [12].

Other feature extraction techniques include segmentation and classification methods, for example, Maximally Stable Extremal Regions [13].

Feature matching is a challenging problem. This step links corresponding points between the data sets. Accuracy of this step determines the quality of transformation model coefficients. Existing algorithms include binary correlation, distance transform, Chamfer matching, dynamic programming, structural matching, chain code correlation, distance of invariant moments [14] and original solutions [15], [16].

Most of the automated GCP studies consider image registration. Spatial resolution of images under consideration is different, but data acquisition process basically is the same. In this study, we consider correction of images obtained from different sources originated from different physical processes.

\section{STUDY SITE AND DATA SETS}

Study site covers the area of the city of Liepāja $\left(56^{\circ} 30^{\prime} 42^{\prime \prime} \mathrm{N}\right.$ $\left.21^{\circ} 00^{\prime} 50^{\prime \prime} \mathrm{E}\right)$, which is the third largest city in Latvia with total area of $60.4 \mathrm{~km}^{2}$, population 70499 (2015) and population density $1037 / \mathrm{km}^{2}[17]$.

Geometric compatibility was tested for data set, including OpenStreetMap data, thermal remote sensing images acquired from Unmanned Aerial Vehicle (UAV) and orthophoto maps in visible light.

\section{OpenStreetMap Data}

OpenStreetMap (OSM) is open geographical data acquired by volunteers using aerial imagery, GPS devices and low-tech maps. OSM data can be downloaded as SHP files. In the given application, only multipolygon layer was used to analyse buildings. More than 8000 buildings were registered in OSM for area covered by remote sensing images.

\section{Thermal Images}

Thermal images were acquired on 11 December 2011 using $\mathrm{HgCdTe}$ sensor in $0.2 \mathrm{~m}$ spatial resolution. Spectral resolution was $8-14 \mathrm{~mm}$. Inertial Measurement Unit (IMU) and GNSS were employed during the flight and data were projected on the plane acquired by radar measurements. Geometrical correction was performed using GCPs selected from building vector maps, but accuracy varied in different parts of the image. Thermal images covered approximately 3200 hectares.

\section{Orthophotomaps}

Orthophotomaps in visible light and with spatial resolution of $0.4 \mathrm{~m}$ were acquired from the Latvian Geospatial Information Agency for year 2015. Images were taken in "leaves-on" season according to requirements of aerial images for countrywide database [18].

Figure 1 shows contours of one sample building in all three data sets. Offsets observed are significant enough to degrade the quality of statistical analysis, especially, when creating raster descriptors used for building polygons.

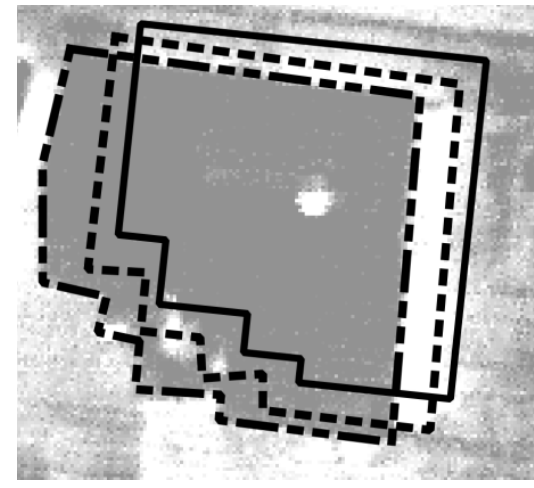

Fig. 1. Contour of the sample building obtained from different data sources. Solid line - OSM data, dotted line - orthophotomap, dashed dot line - thermal image, background layer - thermal image.

\section{METHODOLOGY}

Principal steps of semi-automatic methodology are shown in Fig. 2.

\section{A. Extract Potential GCP Points and Significant Lines from OSM Data}

The polygons of building are selected from OSM data where each polygon is marked with a unique label. If polygon has less than 6 vertices, then four longest lines are found and marked as significant lines. Potential GCP points are corner (crossing) points that are generated by those significant lines. If polygon has more than 6 vertices, then 3 corner points are found where corresponding edges have the longest total 
length. Lines forming 3-corner points are marked as significant lines and corner points are marked as potential GCPs.

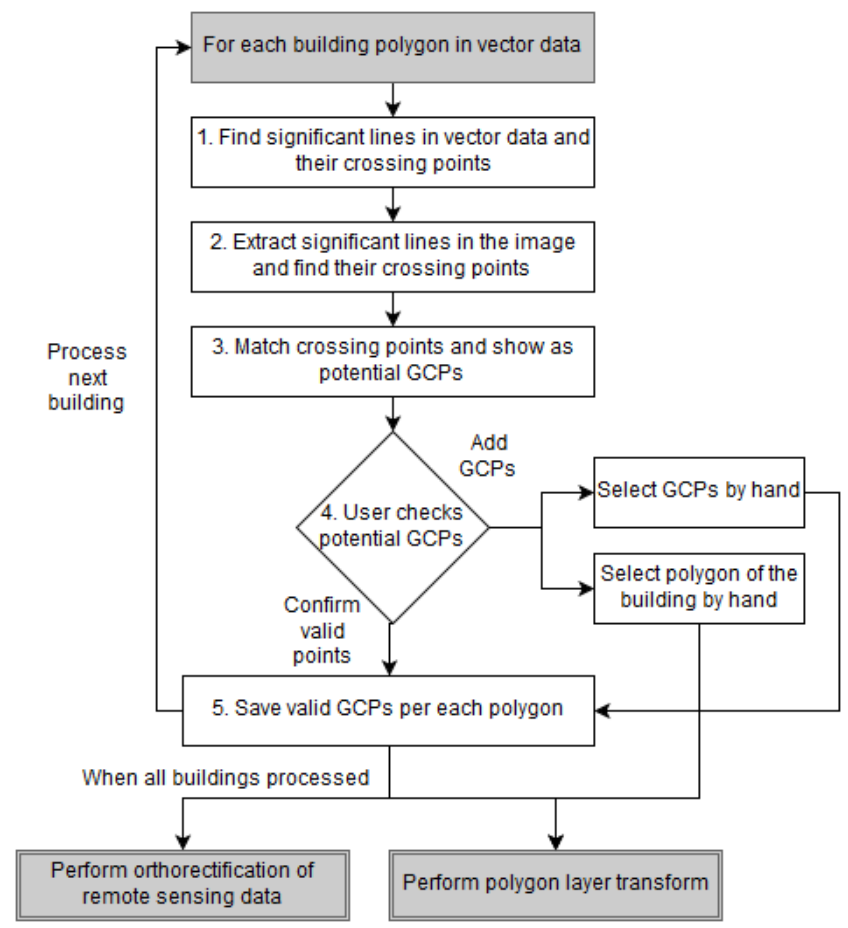

Fig. 2. Basic steps of semi-automatic GCP selection methodology.

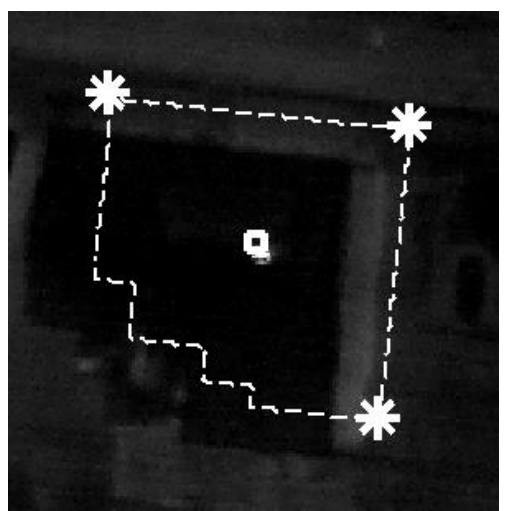

Fig. 3. Building polygon from OSM data shown by dashed line. Potentia GCPs formed by significant lines of the polygon are shown by a white star, polygon centroid is shown by a white square. Background layer - thermal image.

\section{B. Feature Extraction from the Image}

The task is to extract corners of the buildings in the remote sensing images.

Basic idea: extract significant lines forming building contour and link the line crossing points with the building corner points presented in OSM data.

\section{Preprocessing}

The building to be processed is extracted from the whole remote sensing image with surrounding border (neighbourhood) around the expected building location according to OSM data in order to include possible offsets in the extracted image. This step helps discard potential influence of neighbouring buildings. In this study, buffer around the bounding box of building polygon was 30 pixels (6 meters). Noise was reduced by 5 × 5 median filter. Median filter was chosen to prevent smoothing of edges comparing with other popular filters, such as Wiener filtering or Gaussian filter [19].

\section{Extraction of Edges}

Edges were extracted by using Sobel edge detector with a sensitive threshold [20]. Since we are interested only in significant lines that are expected to be formed by strong edges, Sobel edge detector outperforms frequently applied Canny edge detector, which is more sensitive to intensity changes and aims at finding connected edges introducing extra information. In this case, the extraction of significant lines would require additional computational burden.

\section{Extraction of Lines of Interest}

Classical solution for line extraction from binary edges is Hough transform [21]. Its basic implementation requires additional post processing when straight lines are distorted geometrically during image acquisition; therefore, in this study a specific line scanning algorithm was proposed.
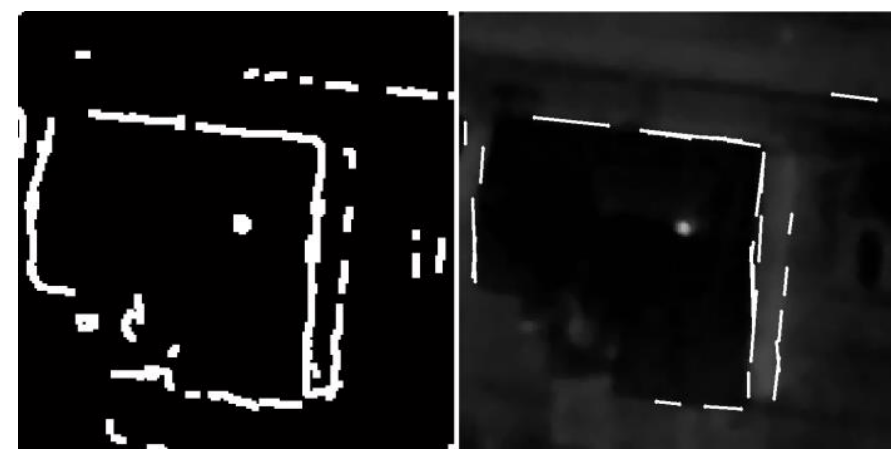

Fig. 4. Image to the left: edges extracted by Sobel edge detector (edges are thickened for illustration purposes). Image to the right: 15 longest lines obtained by Hough transform are shown by white lines.

\section{Line Scanning Method}

Line scanning algorithm in the binary image is based on two assumptions: 1) offset always is so small that centroid of the reference polygon is located inside the area corresponding to the building in the remote sensing image, 2) actual building outer contour lines go approximately in the same direction (same slope) as lines in the reference polygon.

The basic idea of the algorithm is to slide each significant line over the image in perpendicular direction of the line slope and to check overlap between the line and the binary edge image. Location with maximum overlap is the actual location of the line of interest. Thickness of the scanning line is larger than 1 (7 pixels in our study) to account for contour distortions.

Each of the significant lines is defined by a linear function equation:

$$
y_{s}=a x+b,
$$

where $a$-slope and $b$-intersect.

For each of the significant lines it is necessary to find a perpendicular line $y_{p}=a_{p} x_{p}+b_{p}$, which crosses the centroid of the reference polygon:

$$
\begin{gathered}
a_{p}=-1 / a \\
b_{p}=-a_{p} * C_{x}+C_{y},
\end{gathered}
$$


where $C_{x}, C_{y}$ are $x$ and $y$ coordinates of the reference polygon centroid, respectively.

Unique coordinates $\mathrm{y}$ of the perpendicular line $y_{p}$ on the image grid represent intersect points of the significant line $y_{s}$ when it slides over the image.

Line $y_{s}$ is translated over the image as line $y_{t}$ by keeping the same slope $a$, but changing intersect according to unique coordinates y of the line $y_{p}$. Since slope of actual edge can be a bit different from the line $y_{t}$, the translated line is generated with certain thickness (in our case thickness is 7 pixels).

Translation is geometrically constrained. Distance between lines $y_{s}$ and $y_{t}$ cannot exceed certain threshold $T_{d}$ (empirically $T_{d}=20$ was chosen so it would be larger than the largest offset observed in data). In some cases, the offset between building in image and polygon is so large that it was not possible to use constraining translation to only one side of the centroid.

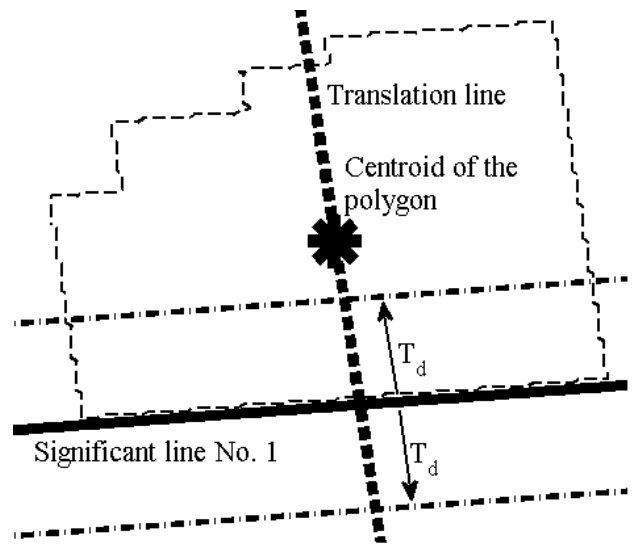

Fig. 4. Translation of the significant line.

Overlap o is calculated between rasterized line $y_{t}$ and binary edge image BW as follows:

$$
o=\sum \sum y_{t} A N D B W
$$

Maximum value of o over the image shows intersect of actual line $y_{s}$ location. However, the line is additionally corrected by linear regression. Image $x$ and $y$ coordinates of white pixels are extracted in maximum overlap area in the image and passed to algorithm for fitting first order polynomial. Slope and intersect of this polynomial shows the actual location of the final line $y_{f}$ in image coordinates. If absolute difference in slopes of $y_{f}$ and $y_{t}$ exceeds certain threshold $T_{s}$ ( $T_{s}=0.2$ in our study), then slope of $y_{t}$ is employed $a_{y f}=a_{y t}$. Intersection points of final lines are found by using standard equations in geometry, for example:

$x_{G C P 1}=\left(b_{f 2}-b_{f 1}\right) /\left(a_{f 1}-a_{f 2}\right)$,

$y_{G C P 1}=a_{f 1} * x_{G C P 1}+b_{f 1}$,

where $x_{G C P 1}$ and $y_{G C P 1}$ are $\mathrm{x}$ and $\mathrm{y}$ coordinates of the first GCP, f1 and f2 label parameters of final significant lines No. 1 and 2 making crossing point GCP1.

Once pixels in binary edge image corresponding to the significant line are found, their locations are replaced with value 0 . Some of significant lines forming the building are almost parallel; therefore, one significant line scan can find actual location of the other significant line. This is not constrained because the order of significant lines does not impact GCP matching.

\section{GCP Matching}

Offsets can be the cause of errors in the results - the obtained Euclidean distances can be small also for wrongly estimated points. In this matter, spatial relationship between points can be used more efficiently than matching by Euclidean distance. This approach can produce four crossing points as maximum (if four significant lines are selected); therefore, points can be labelled by four labels: UL - upper left, UR - upper right, LL - lower left and LR - lower right points. Upper left point has the lowest values of coordinate $\mathrm{y}$ and coordinate $x$; upper right point has the lowest value of coordinate $y$ and the highest value of coordinate $x$, etc. Potential GCPs from OSM data and crossing points of corrected significant lines were marked with these labels. After this step UL point is matched with other UL point, UR point with other UR point and so on.

\section{User Input}

Due to the different possible real life scenarios, this approach remains semi-automatic, meaning that a user must double-check and confirm selection of GCPs and/or provide manual selection in difficult cases. The given implementation includes options to approve only valid GCPs found by an automatic procedure, and allows selecting GCPs manually for every polygon, if necessary, as well as correcting the shape of polygon when reference polygons do not represent the actual shape of the building.

\section{E. Application of GCPs}

Once GCPs are retrieved, they can be applied in two ways. If the further task requires polygon statistics, then it is worth transforming only a polygon image leaving remote sensing images unchanged. If all data sets must be registered in one standard, then geometric transform can be applied to remote sensing images using a polygon image as a reference image.

\section{Polygon Transformations}

Remote sensing images - no geometrical transformation.

Polygon image - affine transform applied to every polygon separately according to GCPs selected.

GCPs are used to compute the affine transformation matrix for a polygon. According to the number of GCPs, affine transform can perform translation, rotation, scaling, shear and reflection [19]. Affine transform is applied to a small polygon image cut out from the original image during a preprocessing step. Since centroid location should not change with respect to polygon edges, it can be used to project back the transformed polygon into the original image. Affine transform can be applied to centroid coordinates as well. Polygon pixels with specific label are deleted (replaced with zeros) and transformed polygon is placed in the big image according to its centroid values. 


\section{Geometric Correction}

Remote sensing images - geometrical transformation using second order polynomial.

Polygon image - no geometrical transformation, reference image.

This is a common geometric correction procedure described extensively in remote sensing literature [1], [2]. GCPs are used to calculate transformation coefficients for a transformation model. The second order polynomial transformation model was evaluated.

\section{Accuracy Assessment}

Accuracy for polygon transformation is expressed as percentage of automatically corrected buildings (a user confirms all points found by an algorithm), percentage of buildings corrected using only a few points found by the methodology (a user deletes wrongly estimated GCPs) and percentage of building polygons corrected by a user manually.

Accuracy for geometric correction is calculated as a root mean square error between the required point location and point locations provided by the transform model:

$$
R M S E=\sqrt{\frac{\sum_{i=1}^{n}\left(\overline{x_{l}}-x_{i}\right)^{2}+\left(\overline{y_{l}}-y_{i}\right)^{2}}{n}},
$$

where $n-$ a number of GCPs;

$x_{i}, y_{i}$ - correct locations of GCPs;

$\overline{x_{l}}, \overline{y_{l}}$ - locations of GCPs found by the transformation model.

In this study, RMSE is expressed in pixel count not metric units.

\section{RESULTS}

\section{A. Polygon Transformations}

For evaluation of the performance of polygon transformation, 100 buildings were selected. The list of buildings was obtained from freely available heat consumption data for year 2011 [22].

Figure 5 shows an example how the provided solution looks like and the resultant polygon transform.
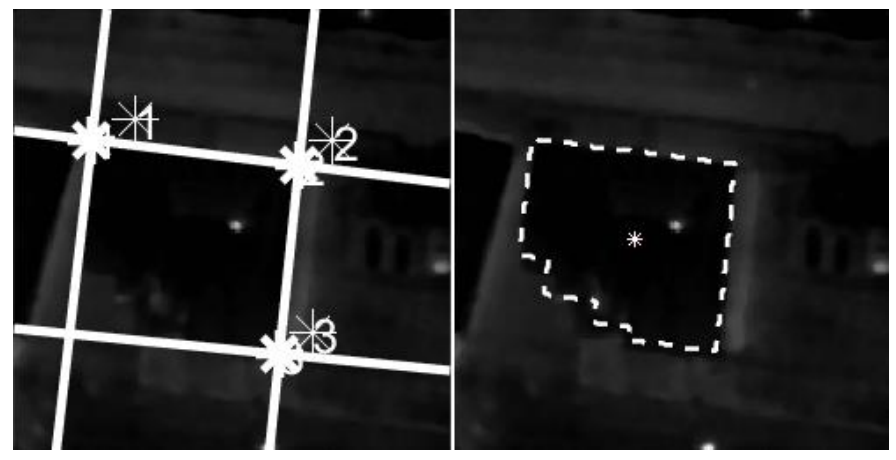

Fig. 5. Image to the left. White lines show significant lines found in the image and the white star marks matched GCPs. Image to the right: a white dotted line shows the transformed polygon contour.
TABLE I

ACCURACY OF AUTOMATICALLY FOUND GCPS

\begin{tabular}{|l|r|r|}
\hline Data & \multicolumn{1}{|c|}{ Thermal } & Orthophotomap \\
\hline GCPs provided & 297 & 309 \\
\hline GCPs found valid & 187 & 230 \\
\hline $\begin{array}{l}\text { Number of polygons transformed } \\
\text { fully }\end{array}$ & $52(52 \%)$ & $35(35 \%)$ \\
\hline $\begin{array}{l}\text { Number of polygons transformed } \\
\text { using partial points }\end{array}$ & $30(30 \%)$ & $20(20 \%)$ \\
\hline Polygons selected by hand & & $45 \%)$ \\
\hline
\end{tabular}

\section{B. Geometric Correction}

Second order polynomial transformation model was applied to subimages with an area of approximately 3 hectares. According to building density, 8-30 GCPs were automatically found and confirmed as valid, but 6-20 GCPs were deleted manually. RMSE for both orthophotomap and thermal image was approximately 0.8 meters with standard deviation of 0.6 meters for GCPs included in the calculation of transformation model. For independent test points (10 selected by hand for each subimage) RMSE was 1 meter with standard deviation of 1.2 meters. Totally RMSE statistics were acquired for 40 subimages.

\section{DISCUSSION AND CONCLUSION}

In this paper, the semi-automatic solution for selecting GCPs in high resolution aerial remote sensing data has been presented. The method requires building polygons (e.g., from OpenStreetMap data) and high resolution remote sensing images as input. Special line scanning algorithm seeks the longest lines in building contours according to polygon data and calculated GCPs. User visually must validate and confirm automatically estimated GCPs or perform manual selection of GCPs in more complex cases where the proposed algorithm is not able to achieve good performance.

This semi-automatic solution allows selecting more GCPs much faster than it is typically done by hand considering each building separately, therefore providing the semi-automation of the process.

GCPs have been applied to transform polygons themselves and to perform classical geometric transform.

For automatic analysis of polygon properties, polygon transformation is recommended and semi-automatic approach can be applied to every single polygon of interest.

Classical geometric transform is recommended for visual assessment and cases where one might decide to correlate remote sensing images with each other.

Accuracy assessment has shown significant improvement in accuracy by using our proposed semi-automatic approach; however, accuracy assessment is affected by subjective reasons for geometric correction. For polygon transform, accuracy is affected by shape complexity of the buildings in test data as well as image acquisition properties.

The main advantage of the methodology provided here is GCP selection speed. Despite the individual manual selection speed of image analysts, confirmation of points takes less time than searching and selecting GCPs by hand solely. 
Automated selection of GCPs requires less human input on orthophotos than thermal images because edges are sharper and contrast between roof and its surroundings is higher than in thermal images.

Automated extraction of significant lines has been affected by following challenges:

- Remote sensing data are acquired by different sensors in different wavelengths, at different view angles and different seasons.

- In some cases, OSM data contain only approximated contour of the building without small details.

- Thermal images contain strong noise component; borders of the objects are smooth and are spatially distorted.

- View angle in both thermal and orthophotomaps produces false corners and walls can be seen from one side.

- Spectral and intensity differences between roofs and other objects are not significantly big.

- In "leaves-on" season, trees occlude some parts of the buildings and shadows cause strong edges.

- In thermal images, high contrast areas are related with heat leaks. In case of heating mains, these high contrast areas can be linear and located near the edges of roofs in the image.

In the future research, the proposed semi-automatic algorithm could be improved to deal with automatic evaluation of selected GCPs. More detailed analysis must be performed to analyse the neighbourhood of GCP locations in the image in order to distinguish between true and false GCPs with even smaller user interaction.

\section{ACKNOWLEDGMENT}

This study has been performed within the framework of Project No. 2/EEZLV02/14/GS/058 "Regional Strategy on Reduction of Greenhouse Gas Emissions from Buildings in Largest Cities in Kurzeme Planning Region, Using Satellite Imaging and State-of-the-Art Thermal Auditing Technologies" that is part of European Economic Area Financial Mechanism 2009-2014 Programme "National Climate Policy" Small Grant Scheme open tender "Capacity Building in the Field of Research and Measures for Enhancing Society's Understanding about Climate Change and its Consequences".

\section{REFERENCES}

[1] P. M. Mather, Computer Processing of Remotely-Sensed Images: an introduction, 3rd ed. John Wiley \& Sons, 2005, 324 p.

[2] J. Wang, Y. Ge, G. B. M. Heuvelink, C. Zhou and D. Brus, "Effect of the sampling design of ground control points on the geometric correction of remotely sensed imagery," International Journal of Applied Earth Observation and Geoinformation, vol. 18, pp.91-100, Aug. 2012. https://doi.org/10.1016/j.jag.2012.01.001

[3] W. Ma, J. Yang, X. Ning and W. Gao, "A quantitative evaluation method of ground control points for remote sensing image registration,' Progress In Electromagnetics Research M, vol. 34, pp. 55-62, 2014. https://doi.org/10.2528/PIERM13092902

[4] O. Benarchid and N. Raissouni, "Support Vector Machines for Object Based Building Extraction in Suburban Area using Very High Resolution Satellite Images, a Case Study: Tetuan, Morocco," IAES International Journal of Artificial Intelligence, vol. 2, iss. 1, March 2013. https://doi.org/10.11591/ij-ai.v2i1.1781

[5] Y. K. Han, Y. G. Byun, J. W. Choi, D. Y. Han and Y. I. Kim, "Automatic registration of high-resolution images using local properties of features," Photogrammetric engineering \& remote sensing, vol. 78 no. 3, pp. 211-221, March 2012. https://doi.org/10.14358/PERS.78.3.211

[6] C. C. Liu and P. L. Chen, "Automatic extraction of ground control regions and orthorectification of remote sensing imagery," Optics express, vol. 17, iss. 10, pp. 7970-7984, 2009. https://doi.org/10.1364/OE.17.007970

[7] L. J. Quackenbush, "A review of techniques for extracting linear features from imagery," Photogrammetric Engineering \& Remote Sensing, vol. 70, no. 12, pp. 1383-1392, Dec. 2004 https://doi.org/10.14358/PERS.70.12.1383

[8] W. Yang, X. Wang, B. Moran, A. Wheaton and N. Cooley, "Efficient registration of optical and infrared images via modified Sobel edging for plant canopy temperature estimation," Computers \& Electrical Engineering, vol. 38, iss. 5, pp. 1213-1221, Sep. 2012. https://doi.org/10.1016/j.compeleceng.2012.05.014

[9] J. Yao, M. R. Ruggeri, P. Taddei and V. Sequeira, "Automatic scan registration using 3D linear and planar features," 3D Research, vol. 1, iss. 6, pp. 1-18, 2010. https://doi.org/10.1007/3DRes.03(2010)06

[10] Y. Ye and J. Shan, "A local descriptor based registration method for multispectral remote sensing images with non-linear intensity differences," ISPRS Journal of Photogrammetry and Remote Sensing, vol. 90, pp. 83-95, Apr. 2014. https://doi.org/10.1016/j.isprsjprs.2014.01.009

[11] L. Yu, D. Zhang and E. J. Holden, "A fast and fully automatic registration approach based on point features for multi-source remotesensing images," Computers \& Geosciences, vol. 34, iss. 7, pp. 838-848, July 2008. https://doi.org/10.1016/j.cageo.2007.10.005

[12] A. Sedaghat, M. Mokhtarzade and H. Ebadi, "Uniform robust scaleinvariant feature matching for optical remote sensing images," IEEE Transactions on Geoscience and Remote Sensing, vol. 49, iss. 11, pp. 4516-4527, Nov. 2011. https://doi.org/10.1109/TGRS.2011.2144607

[13] B. Zitova and J. Flusser, "Image registration methods: a survey," Image and vision computing, vol. 21, iss. 11, pp.977-1000, Oct. 2003. https://doi.org/10.1016/S0262-8856(03)00137-9

[14] S. Khorram, "A feature-based image registration algorithm using improved chain-code representation combined with invariant moments," IEEE Transactions on Geoscience and Remote Sensing, vol. 37, iss. 5, pp. 2351-2362, Sep. 1999. https://doi.org/10.1109/36.789634

[15] H. Sui, C. Xu, J. Liu and F. Hua, "Automatic Optical-to-SAR Image Registration by Iterative Line Extraction and Voronoi Integrated Spectral Point Matching," IEEE Transactions on Geoscience and Remote Sensing, vol. 53, iss. 11, pp.6058-6072, Nov. 2015. https://doi.org/10.1109/TGRS.2015.2431498

[16] Z. Xiong and Y. Zhang, "A novel interest-point-matching algorithm for high-resolution satellite images," IEEE Transactions on Geoscience and Remote Sensing, vol.47, iss. 12, pp.4189-4200, Dec. 2009. https://doi.org/10.1109/TGRS.2009.2023794

[17] Liepājas pilsētas Attīstības programma 2015.-2020. gadam. Esošā situācija. [Online]. Available: http://www.liepaja.lv/upload/attistibas_strategija/pielikums_1_esosa_sit uacija_2013_07_01_2015.pdf. [Accessed: Oct. 1, 2016].

[18] Ortofotokartes vispārīgās prasības un izgatavošanas noteikumi. [Online]. Available: map.lgia.gov.lv/file.php?id=39. [Accessed: Oct. 1, 2016]

[19] R. C. Gonzalez and R. E. Woods, Digital image processing, 2008.

[20] Mathworks. Find edges in intensity image. User Manual, 2016.

[21] N. Aggarwal and W. C. Karl, "Line detection in images through regularized Hough transform," IEEE transactions on image processing, vol. 15 , iss. 3, pp. 582-591, March 2006. https://doi.org/10.1109/TIP.2005.863021

[22] Heat consumption data about Liepaja. [Online]. Available: http://www.liepajasenergija.lv/siltumpaterins/269-maju-siltumenergijaspaterina-salidzinajums-2011-gada-novembris-2012-marts. [Accessed: Oct. 1, 2016].

Linda Gulbe obtained Mg. sc. comp. degree in 2012. She is a Doctoral student of the University of Latvia. She works as a Researcher and Lecturer at Ventspils University College. Research interests include computer processing of remotely sensed data, object and pattern recognition, artificial intelligence. E-mail: linda.gulbe@venta.lv

Gundars Korāts is a Researcher and Lecturer at Ventspils University College. Research interests include signal processing, artificial intelligence, and image processing.

E-mail: gundars.korats@venta.lv 